\title{
Magnetic Reconnection Inflow near the CME/Flare Current Sheet
}

\author{
J. Lin ${ }^{1}$, Y.-K. Ko ${ }^{1}$, L. Sui ${ }^{2}$, J. C. Raymond ${ }^{1}$, G. A. Stenborg ${ }^{2}$, \\ Y. Jiang ${ }^{3}$, S. Zhao ${ }^{3}$, and S. Mancuso ${ }^{1},{ }^{4}$ \\ ${ }^{1}$ Harvard-Smithsonian Center for Astrophysics, 60 Garden Street, Cambridge, MA 02138, \\ USA, email: jlin@cfa.harvard.edu \\ ${ }^{2}$ Department of Physics, Catholic University of America, 620 Michigan Avenue, Washington, \\ DC 20064, USA \\ ${ }^{3}$ National Astronomical Observatories of China/Yunnan Observatory, Chinese Academy of \\ Sciences, P. O. Box 110, Kunming, Yunnan 650011, China \\ ${ }^{4}$ INAF/Osservatorio Astronomico di Torino, 20 Strada Osservatorio, 1-10025 Pino Torinese, \\ Italy
}

\begin{abstract}
This work reports direct observations of the magnetic reconnection site during an eruptive process occurring on November 18, 2003. The event started with a rapid expansion of a few magnetic arcades located over the east limb of the Sun and developed an energetic partial halo coronal mass ejection (CME), a long current sheet and a group of bright flare loops in the wake of the CME. It was observed by several instruments both in space and on ground, including the EUV Imaging Telescope, the Ultraviolet Coronagraph Spectrometer, and the Large Angle and Spectrometric Coronagraph experiment on board the Solar and Heliospheric Observatory, the Reuven Ramaty High Energy Solar Spectroscopic Imager, as well as the Mauna Loa Solar Observatory Mark IV K-coronameter. We combine the data from these instruments to investigate various properties of the eruptive process, including those around the current sheet. The composite of images from different instruments and the corresponding results specify explicitly how the different objects developed by a single eruptive process are related to one another.
\end{abstract}

Keywords. Sun: coronal mass ejections, flares, MHD

\section{Introduction}

The catastrophe model of solar eruptions suggests that as the catastrophe takes place, the closed magnetic field lines in the configuration are stretched so severely that they effectively open up, a long current sheet forms separating two magnetic fields of opposite polarities, and magnetic reconnection invoked in the current sheet creates flare ribbons on the solar surface and flare loops in the corona (e.g., Lin \& Forbes 2000; Priest \& Forbes 2002; Lin et al. 2003).

As a result of the high electrical conductivity and the force-free environment in the corona, the current sheet is confined to a very local and thin region. This makes direct observation of the current sheet extremely difficult (refer to Ko et al. 2003 for the discussions and brief review on this issue). Therefore, information about magnetic reconnection inside the current sheet is usually deduced indirectly by observing the dynamical behaviors of the products of magnetic reconnection, such as the separating flare ribbons on the solar surface (Poletto \& Kopp 1986; Qiu et al. 2004), the growing flare loops in the corona (Sui et al. 2004), and so on. The magnetic reconnection inflow around the X-type neutral point over the flare loops was also reported and used to analyze the properties of magnetic reconnection in an eruptive process (Yokoyama et al. 2001). 
Utilizing the ultraviolet spectroscopic observations from the Ultraviolet Coronagraphic Spectrometer (UVCS) on board the Solar and Heliospheric Observatory (SOHO), together with other remote-sensing data, Ciaravella et al. (2002) and Ko et al. (2003) conducted comprehensive analyses from various aspects on several eruptive processes that clearly manifested both coronal mass ejections (CMEs) and solar flares, and confirmed the existence and development of the long current sheet in the events as predicted by Lin \& Forbes (2000). Webb et al. (2003) surveyed 59 CMEs observed by the Solar Maximum Mission from 1984 to 1989, and found that about half were followed by co-axial, bright rays suggestive of newly formed current sheets.

The event of November 18, 2003 provides us another opportunity to observe directly the current sheet and the associated magnetic reconnection process. It was observed by instruments both in space and on the ground.

\section{Observations and Results}

This event occurred on the east limb of the Sun. No apparent magnetic structure appeared in the nearby region in $\mathrm{H} \alpha$ filtergrams. After having looked at both the magnetograms obtained by the Michelson Doppler Imager (MDI) on board $\mathrm{SOHO}$ and the $\mathrm{H} \alpha$ images obtained on ground within the following three successive days, we find that the eruption took place right between the two active regions, AR0507 and AR0508, located north and south of it, respectively, but its relations to these two active regions are not clear. On the other hand, the initial stage and the subsequent development of the eruption in the lower corona were clearly recorded in $195 \AA$ by the EUV Imaging Telescope (EIT) on board SOHO, and the Reuven Ramaty High Energy Solar Spectroscopic Imager (RHESSI, Lin et al. 2002). The consequences in the higher corona were observed by UVCS and the Large Angle and Spectrometric Coronagraph Experiment (LASCO) on board SOHO, and the Mauna Loa Solar Observatory Mark IV K-coronameter (MLSO MK4).

We were first impressed by this event with the inward motions of the magnetic structures that were severely stretched by the eruption, and we were then impressed with morphological features of the CME developed during the eruption. The initial phase of the eruption at in the low corona observed in $195 \AA$ by EIT is manifested clearly by the movies, the LASCO movie shows the magnetic structures before and after the CME passed through the field of views of LASCO C2 and C3 $\dagger$, and more pictures and detailed discussions can be easily found in our recent work (Lin et al. 2005). The CME velocities at maxima are $1939 \mathrm{~km} / \mathrm{s}$ at the front edge and $1484 \mathrm{~km} / \mathrm{s}$ at the core, respectively.

\section{Discussions Conclusions}

Following Ciaravella et al. (2002), Ko et al. (2003) and Webb et al. (2003), the current sheet developing in the wake of a CME is investigated once again. The event discussed here occurred on the east limb of the Sun on November 18, 2003. It started from 08:48 UT with the fast expansion of a group of sheared arcades. No apparent prominence structure can be recognized in either H $\alpha$ filtergrams or EIT $195 \AA$ images prior to the eruption. This event developed a long and thin current sheet behind the CME. The magnetic reconnection inflow near the current sheet following the arcade expansion was observed, and the corresponding rate of magnetic reconnection $M_{A}$ ranges from 0.01 to 0.23 . The value of $M_{A}$ in reality may not be constant and should cover a wider range.

$\dagger$ Movies can be found at: http://hesperia.gsfc.nasa.gov/ sui/20031118. 
A similar process of magnetic reconnection inflow was also reported by Yokoyama et al. (2001) for the event on March 18, 1999. But the inflow velocity and $M_{A}$ deduced by them are both one order of magnitude smaller than what we obtained in the present case. Another difference between two events lies in the initial configurations. The magnetic arcades in the present case possess a much more compact structure than those of Yokoyama et al. (2001). One of the advantages of this work over that of Yokoyama et al. (2001) is that the reconnection outflow in the present case might be observed. This allows us to estimate $V_{A}$ directly in the reconnection inflow region in a more reliable way without making any extra assumptions. Furthermore, the UVCS observations make it possible for us to avoid unnecessary confusion in measuring the inflow speed of reconnection (e.g., see Chen et al. 2004).

The eruptive process manifests an energetic CME and a bright flare loop system that was covered by a cusp structure. The maximum velocities of the CME leading edge and the core were $1939 \mathrm{~km} \mathrm{~s}^{-1}$ and $1484 \mathrm{~km} \mathrm{~s}^{-1}$, respectively. This event was observed by various instruments from both space and ground. Analyzing the observational data from these instruments yields the conclusion that the CME and the flare are connected by a stretched current sheet in which magnetic reconnection occurs and converts the magnetic energy into heating and kinetic energy. The morphological features of the disrupted magnetic field involved in this event fit those of the cartoon shown in Figure 1 of Lin et al. (2004) very well, which implies that the cartoon depicts the common characteristics of the eruptive processes that give rise to both flare and CME. The magnetic configuration in reality may be much more complex than that shown in the cartoon, but the fundamental physical processes should be the same!

\section{Acknowledgements}

JL is grateful to T. G. Forbes for valuable discussions. This work was supported by NASA under grants NNG04GE84G and NAG5-12827 to the Smithsonian Astrophysical Observatory. The work of YJ and SZ was supported by the National Science Foundation of China under grant 10173023 to the Yunnan Astronomical Observatory. SOHO is a joint mission of the European Space Agency and US National Aeronautics and Space Administration.

\section{References}

Chen, P. F., Shibata, K., Brooks, D. H., \& Isobe, H.: 2004, ApJ, 602, L61.

Ciaravella, A., et al. 2002, ApJ, 575, 1116.

Ko, Y., Raymond, J. C., Lin, J., Lawrence, G., Li, J., \& Fludra, A. 2003, ApJ, 594, 1068.

Lin, J. 2002, Chinese J. Astron. Astrophys., 2, 539.

Lin, J. \& Forbes, T. G. 2000, JGR, 105, 2375.

Lin, J., Raymond, J. C., \& van Ballegooijen, A. A. 2004, ApJ, 602, 422.

Lin, J., Soon, W., \& Baliunas, S. L. 2003, New Astron. Rev., 47, 53.

Lin, R. P., et al., 2002, Solar Phys., 210, 3.

Poletto, G. \& Kopp, R. A.: 1986, in The Lower Atmosphere of Solar Flares, (ed.) D. F. Neidig, NSO, Sunspot, NM, P. 453.

Priest, E. R. \& Forbes, T. G. 2002, A\&A Rev., 10, 313.

Qiu, J., Wang, H., Cheng, C. Z., \& Gary, D. E.: 2004, ApJ, 604, 900.

Sui, L., Holman, G. D., \& Dennis, B.: 2004, ApJ, 612, 546.

Webb, D. F., Burkepile, J., Forbes, T. G., \& Riley, P., 2003, JGR, 108 (A12), 1440.

Yokoyama, T., Akita, K., Morimoto, T., Inoue, K., \& Newmark, J. 2001, ApJ, 546, L69. 